\title{
Losartan attenuates renal interstitial fibrosis and tubular cell apoptosis in a rat model of obstructive nephropathy
}

\author{
PING HE, DETIAN LI and BEIRU ZHANG
}

\begin{abstract}
Department of Nephrology, Shengjing Hospital of China Medical University, Shenyang, Liaoning 110004, P.R. China
\end{abstract}
Received October 16, 2013; Accepted April 16, 2014

DOI: $10.3892 / \mathrm{mmr} .2014 .2304$

\begin{abstract}
Ureteral obstruction leads to renal injury and progresses to irreversible renal fibrosis, with tubular cell atrophy and apoptosis. There is conflicting evidence concerning whether losartan (an angiotensin II type I receptor antagonist) mitigates renal interstitial fibrosis and renal tubular epithelial cell apoptosis following unilateral ureteral obstruction (UUO) in animal models. The aim of this study was to investigate the effect and mechanism of losartan on renal tubular cell apoptosis and renal fibrosis in a rat model of UUO. The rats were subjected to UUO by ureteral ligation and were treated with dimethyl sulfoxide (control) or losartan. The controls underwent sham surgery. The renal tissues were collected 3, 5, 7 and 14 days after surgery for measurement of various indicators of renal fibrosis. UUO increased the expression levels of $\alpha$-smooth muscle actin and collagen I, and the extent of renal tubular fibrosis and apoptosis in a time-dependent manner. Losartan treatment partially attenuated these responses. Progression of renal interstitial fibrosis was accompanied by phosphorylation of signal transducer and activator of transcription 3 (STAT3) and altered the expression levels of two apoptosis-related proteins ( $\mathrm{Bax}$ and $\mathrm{Bcl} 2$ ). Losartan treatment also partially attenuated these responses. The results indicated that losartan attenuated renal fibrosis and renal tubular cell apoptosis in a rat model of UUO. This effect appeared to be mediated by partial blockage of STAT3 phosphorylation.
\end{abstract}

\section{Introduction}

Chronic kidney disease (CKD) results from various types of insult to the kidney and affects $\sim 10 \%$ of the general population worldwide (1). CKD is a progressive process that is characterized by increasing levels of interstitial fibrosis $(1,2)$. Renal

Correspondence to: Dr Ping He, Department of Nephrology, Shengjing Hospital of China Medical University, 36 Sanhao Street, Shenyang, Liaoning 110004, P.R. China

E-mail: doctorhe@126.com

Key words: losartan, unilateral ureteral obstruction, renal interstitial fibrosis, apoptosis, signal transducer and activator of transcription 3 tubulointerstitial fibrosis is the final common step in all forms of end-stage renal disease, and is characterized by aberrant activation and growth of renal fibroblasts $(1,2)$. The activated fibroblast is considered a myofibroblast and is defined by phenotypic changes, including expression of $\alpha$-smooth muscle actin $(\alpha$-SMA) and increased production of fibronectin and other extracellular matrix (ECM) components (1-3). Elucidation of the molecular events responsible for the activation of renal fibroblasts may lead to novel approaches for the treatment of progressive renal diseases.

Unilateral ureteral obstruction (UUO) is characterized by progressive renal atrophy, renal interstitial fibrosis and renal tubular cell apoptosis (4). Numerous molecules and signaling pathways are associated with activation of renal interstitial fibroblasts (2,5). Signal transducer and activator of transcription 3 (STAT3) is highly expressed in renal interstitial fibroblasts following obstructive injury (6) and phosphorylation of this protein mediates renal tubular cell apoptosis following oxidant and ischemia-reperfusion injuries $(7,8)$. There are seven proteins in the STAT family (STAT1-4, STAT5a, STAT5b and STAT6) and these proteins all mediate various cellular functions, including cell survival and proliferation $(9,10)$. A previous study reported increased levels of STAT3 activation by phosphorylation at tyrosine-705 in interstitial fibroblasts of a fibrotic kidney following UUO (6). However, the function of STAT3 in the activation of renal fibroblasts and apoptosis of renal tubular cells has not yet been examined in vivo.

Obstruction of the upper urinary tract has deleterious effects on the kidney and is a significant cause of renal insufficiency in children and adults (11). Even with timely surgical management of urinary tract obstruction, there may still be irreversible damage to the affected renal unit (12). Thus, pharmacological management of obstructive nephropathy is also important. The renin-angiotensin system (RAS) is activated in the developing kidney during normal renal development and is also activated by UUO (3). There is doubt whether RAS blockade mitigates renal interstitial fibrosis and renal tubular epithelial cell apoptosis (12-15).

The present study evaluated the therapeutic effect of losartan on the progression of renal fibrosis and tubular cell apoptosis in a rat model of kidney fibrosis. Furthermore, the effect of losartan on the activation of the STAT3 signaling pathway, a crucial event that occurs during the development of progressive renal fibrosis and tubular cell apoptosis, was investigated. 


\section{Materials and methods}

UUO model and losartan treatment. Male Sprague-Dawley rats (180-220 g) were housed at the Experimental Animal Center of Shengjing Hospital (China Medical University, Shenyang, China) at a constant temperature $\left(18 \pm 1^{\circ} \mathrm{C}\right)$ and under a consistent light/dark cycle (light from 07:00 to 18:00). Food and water were provided ad libitum. The animal protocol was approved by the Animal Care and Use Committee of Shengjing Hospital.

Following induction of general anesthesia by intraperitoneal injection of pentobarbital (50 mg/kg body weight), the abdominal cavity was exposed via a midline incision and the left ureter was isolated and ligated. The sham-surgery animals underwent the same surgical procedures but without ureter ligation. To establish the time course of renal fibrogenesis in this model, four groups of rats (10 rats per group) were sacrificed 3, 5, 7 and 14 days after the ureter ligation. The animals with UUO-induced nephropathy and the sham-surgery animals were administered water ( $5 \mathrm{ml}$ per day) or water supplemented with losartan (30 mg/kg per day). Losartan was dissolved in the water and administered by gavage. The dose of losartan was based on those of previous studies (16-18). At the end of the experiments, all rats were anaesthetized by intraperitoneal administration of ketamine $(75 \mathrm{mg} / \mathrm{kg})$ and xylazine $(10 \mathrm{mg} / \mathrm{kg})$ and kidneys were removed for western blot analysis or histological examination.

Renal morphology. Following fixation in $4 \%$ buffered formalin, the renal tissue was dehydrated through a graded ethanol series, embedded in paraffin and sectioned transversely with a thickness of $4 \mu \mathrm{m}$. Sections were prepared on a microtome and stained with Masson's trichrome. The renal morphology was observed by light microscopy (Olympus, Tokyo, Japan) and the severity of the renal fibrosis was determined by the counting of Masson's trichrome-positive areas. Blue granular and linear deposits were considered positive for collagen staining. Quantification of staining employed a computerized image-analysis program (MetaMorph/C-5050/BX41; Olympus, Tokyo, Japan), and the results are expressed as the percentage of area that was stained.

Immunoblot analysis. Tissue samples were washed three times with phosphate-buffered saline (PBS) and a suspension was prepared with a tissue homogenizer in radioimmunoprecipitation assay buffer (Biyuntian Biosynthesis Biotechnology Co., Ltd., Shanghai, China). Subsequently, the samples were centrifuged at $10,000 \mathrm{x} \mathrm{g}$ at $4^{\circ} \mathrm{C}$ for $20 \mathrm{~min}$, and the supernatants were stored at $-80^{\circ} \mathrm{C}$ until required. The protein concentrations were determined by the bicinchoninic acid protein method (Biyuntian Company) and each sample was adjusted to a protein content of $40 \mu \mathrm{g}$. Subsequently, the samples were denatured in loading buffer [62 mM Tris, $10 \%$ glycerol, $2 \%$ sodium dodecyl sulfate (SDS), $0.003 \%$ bromophenol blue, $\mathrm{pH}$ 7.4] and separated by electrophoresis on an $8 \%$ SDS-polyacrylamide gel $(100 \mathrm{~V}$ for $120 \mathrm{~min})$. Following the electrophoresis, the proteins were transferred to polyvinylidene difluoride-plus membranes (MSI, Westborough, MA, USA; $80 \mathrm{~V}$ for $60 \mathrm{~min}$ ) with a semi-dry protein transfer system (UVP Inc., Upland, CA, USA). The membranes were blocked with $5 \%$ non-fat dry milk or 5\% bovine serum albumin in Tris-buffered saline (TBS; pH 7.4) with $0.21 \%$ Tween-20 (TBST) for $1 \mathrm{~h}$ at room temperature, and washed three times (10 min each) in TBST. The membranes were then incubated with one of the following primary antibodies overnight at $4^{\circ} \mathrm{C}$ in TBST: Anti-STAT-3 and phospho-STAT-3 (p-STAT-3) (Cell Signaling Technology, Inc., Danvers, MA, USA; diluted to 1:1,000), anti-bax and Bcl-2 (Santa Cruz Biotechnology, Inc., Santa Cruz, CA, USA; diluted to 1:500), and anti- $\alpha$-SMA and type I collagen (Sigma, St. Louis, MO, USA; diluted to 1:1,000). The membranes were washed three times (10 min each) in TBST, and then incubated with a peroxidase-conjugated IgG goat anti-rabbit secondary antibody (Kangcheng, Shanghai, China) for $2 \mathrm{~h}$ at room temperature. The membranes were then washed three times (10 min each) in TBST. Visualization was performed using an enhanced chemiluminescence kit (Amersham Pharmacia Biotech Inc., Piscataway, NJ, USA). Each membrane was also probed with an antibody against $\beta$-actin (Santa Cruz Biotechnology, Inc.; diluted to 1:2,000), a loading control. The protein expression levels were quantified by scanning densitometry using a bio-image analysis system (Bio-Rad, Baltimore, MD, USA).

Terminal deoxynucleotidyl transferase-mediated dUTP nick end labeling (TUNEL) assay. The TUNEL assay (In Situ Cell Detection Death kit, POD; Roche Diagnostics, Mannheim, Germany) was used to determine the number of nuclei with fragmented DNA, i.e. the number of apoptotic cells. The tissue sections were deparaffinized and hydrated, and washed three times in PBS for 10 min each. Endogenous peroxidase was quenched for $30 \mathrm{~min}$ with $0.3 \%$ hydrogen peroxide in methanol. The sections were treated with $10 \mu \mathrm{g} / \mathrm{ml}$ proteinase-K for $15 \mathrm{~min}$. Subsequently, the sections were washed with PBS, incubated with deoxynucleotidyl transferase and digoxigenin-dNTP for $1 \mathrm{~h}$, and the reaction was stopped with a termination buffer. The slides were then washed with PBS, and peroxidase-conjugated anti-digoxigenin antibody was added at room temperature for $30 \mathrm{~min}$. Following three washes in PBS, the slides were developed with 3,3'-diaminobenzidine and counterstained with $10 \%$ hematoxylin. Apoptotic nuclei were scored by manual counting of the number of them in 10 fields at $x 400$ magnification. The number of apoptosis-positive cells in the tubules and interstitium were added together for the reported totals. In each group, the apoptotic nuclei were approximately equally distributed among the tubular and interstitial cells.

Statistical analysis. All data are expressed as the mean \pm standard deviation. Statistical analysis was conducted using the Statistical Package for the Social Sciences software, version 10.5 (SPSS, Inc., Chicago, IL, USA). A one-way analysis of variance with Dunnett's multiple comparison test was used to compare mean values. $\mathrm{P}<0.05$ was considered to indicate a statistically significant difference.

\section{Results}

UUO increases TUNEL staining and the expression levels of type I collagen and $\alpha$-SMA. The expression levels of $\alpha$-SMA in the kidneys of the control and model rats were examined 
(Fig. 1). $\alpha$-SMA protein is a marker for tubulointerstitial myofibroblasts, which are responsible for the majority of the interstitial collagen deposition following UUO. Western blot analysis indicated that the kidneys of the control rats had negligible expression levels of $\alpha$-SMA, but that the expression levels of $\alpha$-SMA in the UUO rats were $\sim 3$-fold greater on day three and $\sim 9$-fold greater on day 14 compared with those of the sham rats (Fig. 1A and B). The expression levels of collagen exhibited a similar pattern, although the magnitude of the changes was not as great (Fig. 1A and B).

The extent of tubulointerstitial fibrosis following UUO in renal cortical sections was also examined by use of Masson's trichrome stain. The results indicated that the normal kidneys had collagen in the Bowman's capsule, perivascular adventium and renal capsule. The kidneys from the UUO rats had a notably higher number of Masson's trichrome-positive regions in the tubulointerstitial regions on day three compared with that in the sham rats, and this damage increased over time (data not shown).

The TUNEL staining experiments indicated that apoptotic tubular cells were rare in sections of the kidneys from the sham (control) rats (Fig. 1C). However, the levels of renal tubular apoptosis increased three days after UUO compared with those in the sham rats, and the number of TUNEL-positive cells continued to increase over time (Fig. 1C and D).

Losartan inhibits phosphorylation of STAT3 in the obstructed kidney. Previous studies have indicated increased levels of phosphorylated (p)-STAT3 in the interstitial fibroblasts of fibrotic kidneys $(2,6)$. In agreement with the results of these previous studies, the results of the present study indicated that the levels of p-STAT3 increased over time in the kidneys subjected to UUO (Fig. 2A and B) and were $~ 9$-fold greater than those of the control on day 14. Subsequently, the effect of losartan on STAT3 expression and phosphorylation levels in the kidneys subjected to UUO was examined. The results indicated that the losartan treatment significantly reduced the levels of UUO injury-induced phosphorylation of STAT3, although not to the levels of the control (sham) rats (Fig. 2C and D). Although the UUO injury also induced increased expression levels of STAT3 compared with those in the sham rats, losartan had no effect on the overall STAT3 expression levels (Fig. 2C and D).

Losartan inhibits progression of renal fibrosis. The effect of losartan on interstitial fibrosis was assessed by use of Masson's trichrome staining (Fig. 3A and B). The sham kidneys had tubular lumens that were not dilated, epithelial cells that were cuboidal in shape, no apparent interstitium, and tubules that were back-to-back. However, the kidneys subjected to UUO had significant levels of blue staining by day seven compared with those in the sham rats, indicating interstitial fibrosis, and the tubules were separate from one another. Treatment with losartan significantly reduced the fibrosis, but not to the levels of the control. Quantitation of these results indicated that the interstitial volume was $1.5 \%$ $( \pm 0.5)$ in the control kidneys, $15.7 \%( \pm 3.2)$ in the kidneys subjected to UUO, and $10.1 \%( \pm 1.8)$ in the kidneys of the animals with UUO and losartan treatment (Fig. 3B).
A

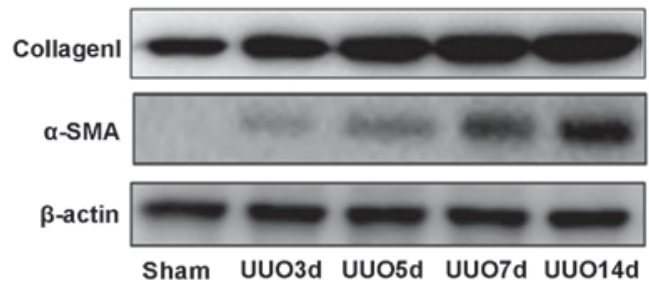

B
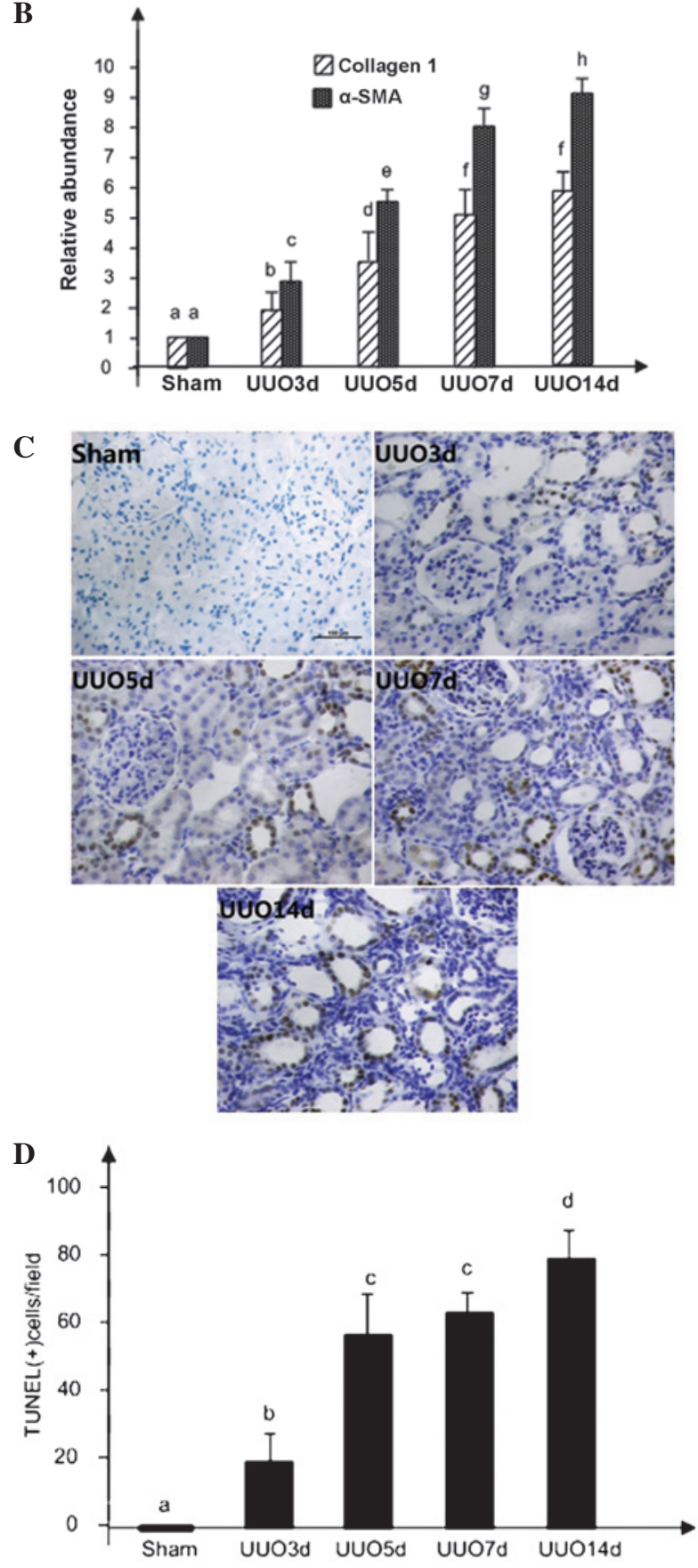

Figure 1. Effect of UUO on the expression levels of renal type I collagen and $\alpha$-SMA in the kidneys of rats subjected to UUO. (A and B) Effect of UUO on the expression levels of renal type I collagen and $\alpha$-SMA from day 3 to 14 ( $\beta$-actin served as a loading control). (C and D) Effect of UUO on the levels of renal tubular apoptosis (TUNEL assay). (C) Representative photomicrographs (magnification, $\mathrm{x} 400$ ) of TUNEL staining in the control kidneys and the treatment kidney tissue at various time periods after UUO. (D) Quantitation of the TUNEL staining results in (C). Values are presented as the mean \pm standard deviation of three independent experiments, and bars with different letters (a-h) are significantly different from one another $(\mathrm{P}<0.05)$. $\alpha$-SMA, $\alpha$-smooth muscle actin; UUO, unilateral ureteral obstruction; TUNEL, terminal deoxynucleotidyl transferase dUTP nick end labeling. 
A

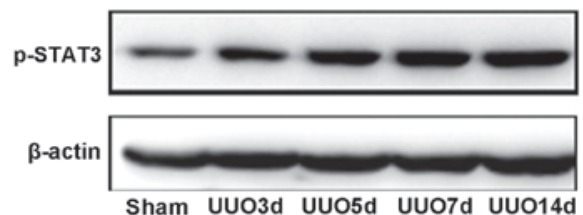

B

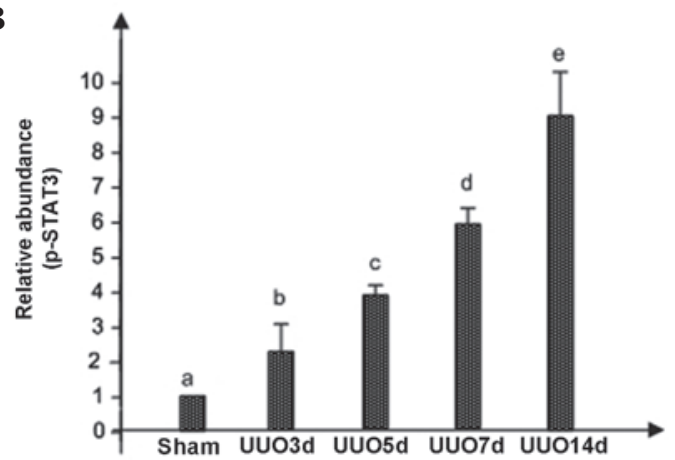

C

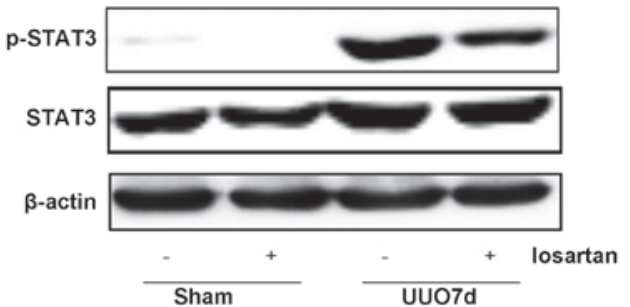

D

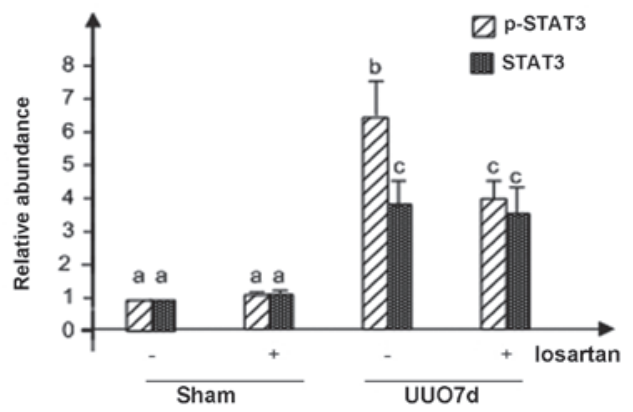

Figure 2. Effect of UUO on the phosphorylation levels of STAT3. (A) Immunoblot analysis of the kidney tissue lysates with antibodies against p-STAT3 or $\beta$-actin (loading control). (B) Quantitation of the western blotting results in (A) by densitometry, expressed as fold-increase over the controls. (C) Effect of losartan on the expression and phosphorylation levels of STAT3 following UUO. (D) Quantitation of the western blotting results in (C) by densitometry, expressed as fold-increase over the controls. Values are the mean \pm standard deviation of three independent experiments, and bars with different letters $(a-h)$ are significantly different from one another $(\mathrm{P}<0.05)$. p-STAT3, phosphorylated signal transducer and activator of transcription 3; UUO, unilateral ureteral obstruction.

The expression levels of type I collagen and $\alpha$-SMA in the kidneys of the control and losartan-treated animals were also assessed (Fig. 3C and D). The results indicated that the expression levels of type I collagen and $\alpha$-SMA were significantly greater in the obstructed kidneys compared with those in the sham rats, and that this effect was partially attenuated by the losartan treatment.

Losartan inhibits tubular apoptotic cell death and apoptotic protein expression in UUO. There is a debate regarding
A

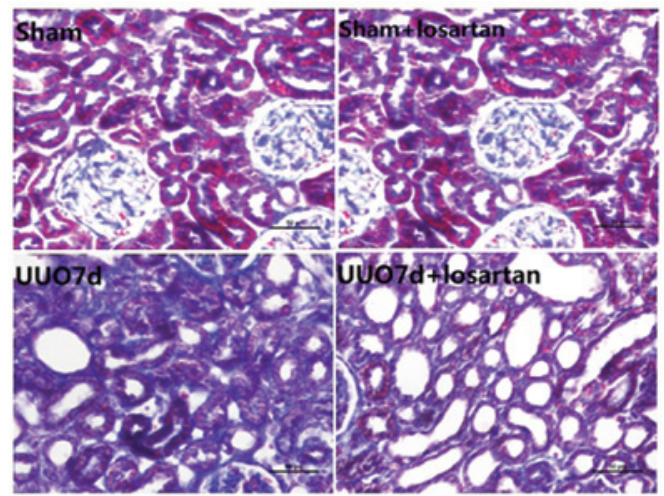

B

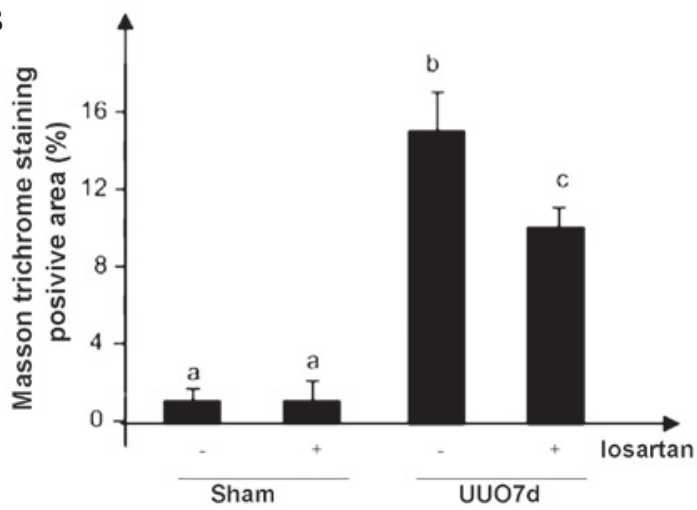

C

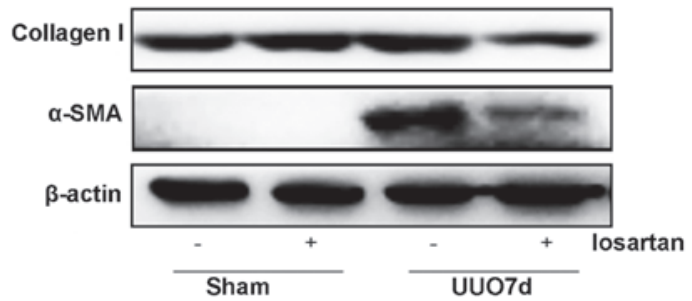

D

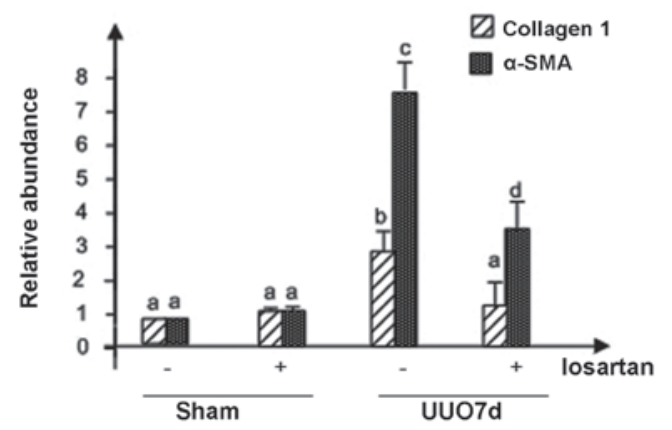

Figure 3. Effect of losartan on renal fibrosis and the expression levels of $\alpha$-SMA and type I collagen following UUO. (A) Representative photomicrographs (magnification, x400) of Masson's trichrome staining of the sham kidneys, and UUO-injured kidneys of rats with and without losartan treatment. (B) Quantitation of Masson trichrome staining results based on 10 random cortical fields. (C) Kidney tissue lysates were subjected to immunoblot analysis with antibodies against $\alpha$-SMA, type I collagen or $\beta$-actin (loading control). (D) Quantitation of the western blot analysis results. Values are presented as the mean \pm standard deviation of three independent experiments, and bars with different letters (a-h) are significantly different from one another $(\mathrm{P}<0.05)$. UUO, unilateral ureteral obstruction; $\alpha$-SMA, $\alpha$-smooth muscle actin.

whether losartan mitigates renal tubular epithelial cell apoptosis following UUO. In particular, certain studies of the effect of type 1 and 2 angiotensin II receptors on apoptosis and renal 

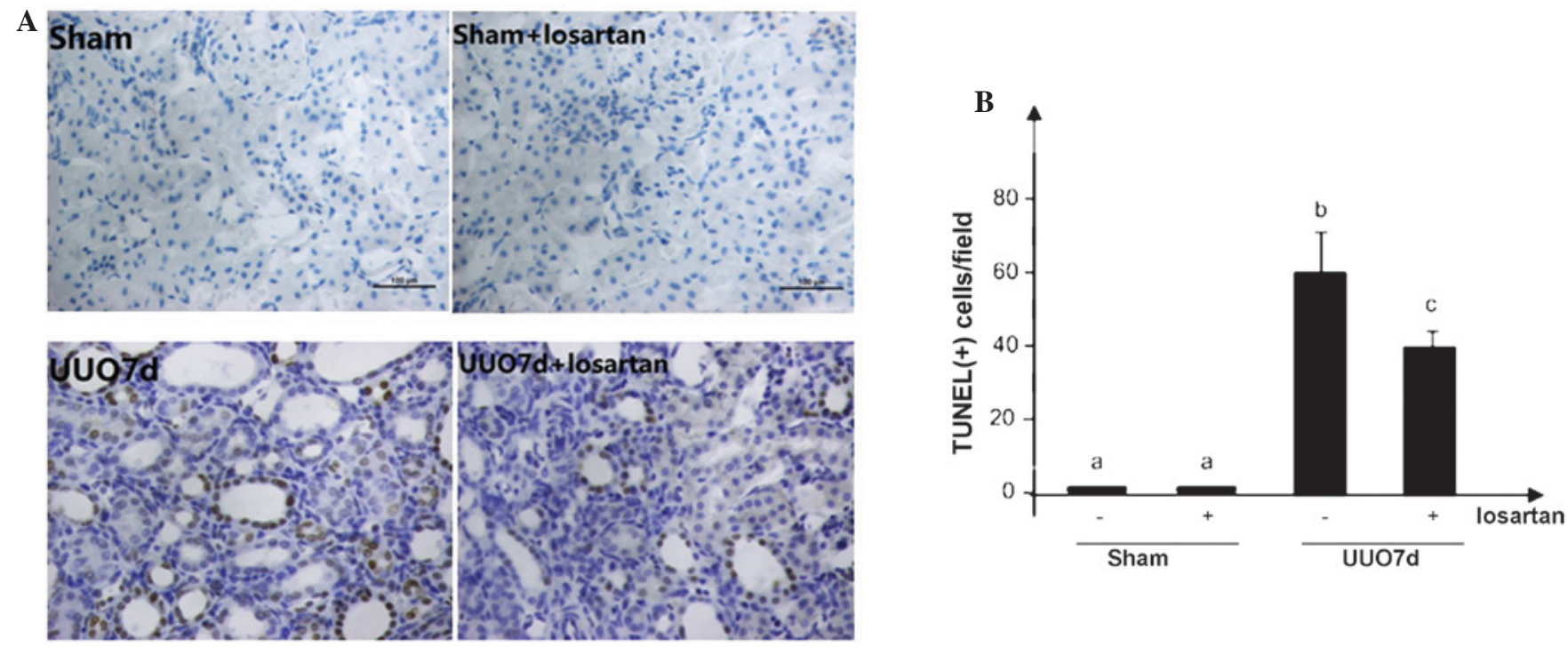

C
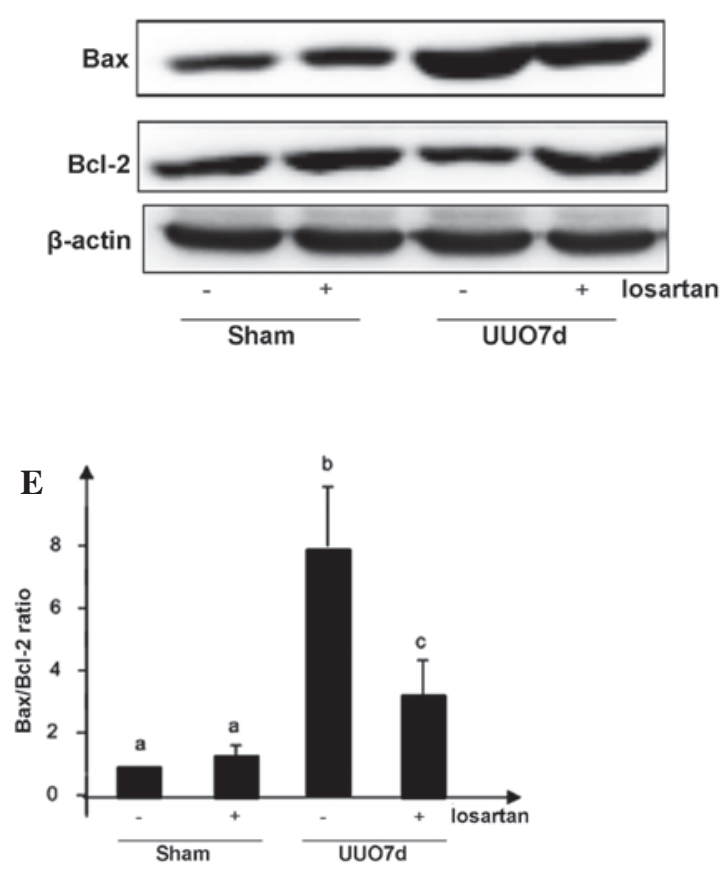

cell proliferation in rats with UUO have reported contradictory results $(15,19,20)$. Thus, TUNEL staining was used in the present study to monitor the levels of apoptotic cell death in the sham-surgery and UUO-injured kidneys with and without losartan treatment (Fig. 4).

There was no evidence of apoptotic cell death in the kidneys subjected to the sham surgery. However, there were a large number of TUNEL-positive cells in the renal tubules at seven days after UUO compared with those in the sham rats. Administration of losartan partially attenuated the effect of UUO on the apoptosis of tubule cells. The expression levels of two important proteins associated with apoptosis, Bcl-2 (an apoptosis inhibitor) and Bax (an apoptosis promoter), were also analyzed by western blot analysis (Fig. 4C and D). The expression levels of renal $\mathrm{Bcl}-2$ were marginally lower and the expression levels of Bax were higher in response to

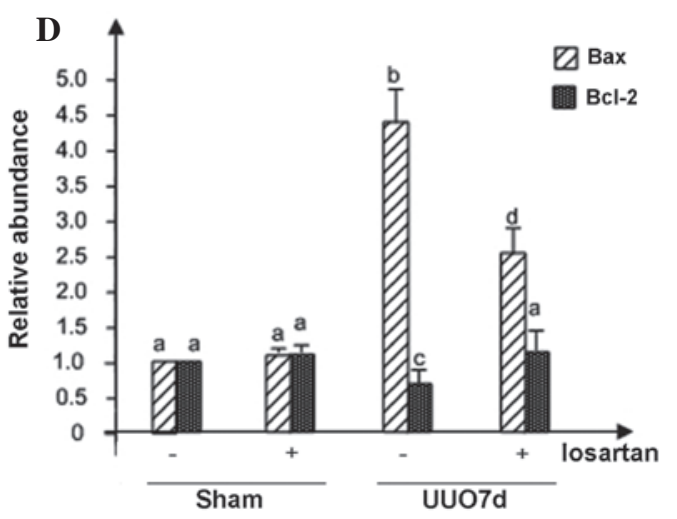

Figure 4. Effect of losartan on the levels of renal tubular cell apoptosis following UUO injury. (A) Representative photomicrographs (magnification, $\mathrm{x} 400$ ) of apoptosis in the sham kidneys with and without losartan and kidneys subjected to UUO with and without losartan. (B) Quantitation of the TUNEL staining results (mean \pm standard deviation of four groups). (C) Immunoblot analysis of the whole kidney tissue lysates with antibodies against Bax, Bcl-2 or $\beta$-actin. (D) Quantitation of the western blot analysis results in (C). (E) The elevated $\mathrm{Bax} / \mathrm{Bcl}-2$ ratio. Values are presented as the mean \pm standard deviation of three independent experiments, and bars with different letters $(\mathrm{a}-\mathrm{h})$ are significantly different from one another $(\mathrm{P}<0.05)$. UUO, unilateral ureteral obstruction; TUNEL, terminal deoxynucleotidyl transferase dUTP nick end labeling.

UUO compared with those in the sham rats. These results are also expressed in a plot of the $\mathrm{Bax} / \mathrm{Bcl}-2$ ratio, a common molecular marker used to assess damage in renal disease and other pathologies (Fig. 4E).

\section{Discussion}

UUO is characterized by progressive renal atrophy, renal interstitial fibrosis and tubular cell apoptosis (4). The levels of RAS activity are increased during the early stages of renal injury, and are further enhanced by UUO (13). The results of the present study indicate that losartan inhibits the increase in renal fibrosis that follows UUO, as indicated by reduced deposition of ECM and expression of type I collagen. However, the mechanism by which losartan attenuates renal fibrosis is not fully understood. A previous study indicated that 
$\alpha$-SMA-positive myofibroblasts are the principal effector cells responsible for ECM overproduction in the fibrotic kidney (2); thus, the present study examined the effect of losartan on their activation in vivo. The data of the present study showed that losartan administration attenuated the interstitial fibrosis and expression levels of $\alpha$-SMA and type I collagen following UUO.

Progression of renal fibrosis is a complicated process that is associated with the concerted interaction of numerous cellular and molecular factors $(2,21)$. Signaling through the Janus kinase (JAK)/STAT pathway is an important component of the response of kidneys to injury and the progression of certain renal diseases. The current model of JAK/STAT signaling holds that binding of cytokines or growth hormones to their receptors activates JAK, which in turn phosphorylates the intracellular domain of the receptor and allows the recruitment and phosphorylation of a STAT (22). Phosphorylated STATs form homo- or hetero-dimers, translocate to the nucleus and transactivate the expression of target genes (22). Previous studies have reported that activation of the STAT3 signaling pathway is involved in the development of renal fibrosis in the UUO experimental model $(6,23)$. In the present study, it was demonstrated that UUO induced STAT3 phosphorylation in the fibrotic kidney by day three and that the levels of p-STAT3 continued to increase until day 14. This marked and persistent increase in the levels of p-STAT3 suggests the importance of this protein in mediating the activation of renal interstitial fibroblasts and progression of renal fibrosis following UUO. The data also clearly demonstrated that administration of losartan partially attenuated the UUO injury-induced phosphorylation of STAT3 and renal fibrosis.

Ureteral obstruction leads to renal damage, and tubular cell apoptosis occurs early, followed by the release of cytokines and chemokines from the injured tubular cells and culminating in inflammatory reactions and interstitial fibrosis (24). Thus, inhibition of the initial tubular cell apoptosis may limit the generation of signals from dying cells, thereby preventing the renal accumulation of proapoptotic and profibrotic cytokines and the subsequent inflammatory response (25). The present study also demonstrated that the renal injury induced by UUO increased the $\mathrm{Bax} / \mathrm{Bcl}-2$ ratio (a proapoptotic signal) in the kidneys compared with in the sham rats, and that prolongation of the UUO injury led to increasing levels of renal tubular cell apoptosis and atrophy. It was also found that losartan partially inhibited the apoptosis associated with unilateral obstructive nephropathy. This observation is in disagreement with a previous study which reported that losartan had no effect on renal cell apoptosis in a murine model of unilateral obstructive nephropathy (26). However, the results of the present study corroborate the findings of Manucha et al (18), which showed that losartan reduced the Fas ligand expression levels in the obstructed kidneys of rats, suggestive of an anti-apoptotic effect. The apoptosis results of the present study are also confirmed by the demonstration of the effect of losartan on STAT3 signaling.

The incidence and prevalence of CKD is increasing worldwide and the primary aims of treatment are to prevent or slow disease progression (27). Progressive renal interstitial fibrosis is a predominant pathological feature of obstructive nephropathy and is considered a common final step of almost all forms of CKD. The findings of the present study suggest that p-STAT3 may be essential in mediating the development of renal interstitial fibrosis in CKD that is caused by different renal diseases. This study also demonstrated that anti-angiotensin treatment with losartan reduced the levels of interstitial fibrosis and apoptosis in a rat model. These results provide novel evidence concerning the role of angiotensin receptor antagonists in slowing the progression of CKD. The results demonstrated that losartan is effective in reducing the levels of interstitial fibrosis and apoptosis in a rat model of unilateral obstructive nephropathy. The antifibrotic effect of losartan is associated with inhibition of the STAT3 signaling pathway, which contributes to renal fibrosis and tubular cell apoptosis. Additional studies are required to test this effect in humans, but the results of the present study are consistent with the view that angiotensin receptor blockade should be considered as the first-choice therapy for patients with chronic obstructive nephropathy.

\section{Acknowledgements}

This study was supported by the Education Commission of Liaoning Province of China (grant no. L2013295) and the National Natural Science Foundation of China (grant no. 81200084).

\section{References}

1. Neilson EG: Mechanisms of disease: fibroblasts - a new look at an old problem. Nat Clin Pract Nephrol 2: 101-108, 2006.

2. Wynn TA: Cellular and molecular mechanisms of fibrosis. J Pathol 214: 199-210, 2008.

3. Liu Y: Epithelial to mesenchymal transition in renal fibrogenesis: pathologic significance, molecular mechanism, and therapeutic intervention. J Am Soc Nephrol 15: 1-12, 2004.

4. Miyajima A, Chen J, Lawrence C, et al: Antibody to transforming growth factor-beta ameliorates tubular apoptosis in unilateral ureteral obstruction. Kidney Int 58: 2301-2313, 2000.

5. Grande MT and López-Novoa JM: Fibroblast activation and myofibroblast generation in obstructive nephropathy. Nat Rev Nephrol 5: 319-328, 2009.

6. Kuratsune M, Masaki T, Hirai T, et al: Signal transducer and activator of transcription 3 involvement in the development of renal interstitial fibrosis after unilateral ureteral obstruction. Nephrology (Carlton) 12: 565-571, 2007.

7. Arany I, Megyesi JK, Nelkin BD and Safirstein RL: STAT3 attenuates EGFR-mediated ERK activation and cell survival during oxidant stress in mouse proximal tubular cells. Kidney Int 70: 669-674, 2006.

8. Yang N, Luo M, Li R, et al: Blockage of JAK/STAT signalling attenuates renal ischaemia-reperfusion injury in rat. Nephrol Dial Transplant 23: 91-100, 2008

9. Stepkowski SM, Chen W, Ross JA, et al: STAT3: an important regulator of multiple cytokine functions. Transplantation 85: 1372-1377, 2008

10. Turkson J and Jove R: STAT proteins: novel molecular targets for cancer drug discovery. Oncogene 19: 6613-6626, 2000.

11. Yeh $\mathrm{CH}$, Chiang HS, Lai TY and Chien CT: Unilateral ureteral obstruction evokes renal tubular apoptosis via the enhanced oxidative stress and endoplasmic reticulum stress in the rat. Neurourol Urodyn 30: 472-479, 2011.

12. Jones EA, Shahed A and Shoskes DA: Modulation of apoptotic and inflammatory genes by bioflavonoids and angiotensin II inhibition in ureteral obstruction. Urology 56: 346-351, 2000.

13. Coleman CM, Minor JJ, Burt LE, et al: Angiotensin AT1-receptor inhibition exacerbates renal injury resulting from partial unilateral ureteral obstruction in the neonatal rat. Am J Physiol Renal Physiol 293: F262-F268, 2007.

14. Ishidoya S, Morrissey J, McCracken R, et al: Angiotensin II receptor antagonist ameliorates renal tubulointerstitial fibrosis caused by unilateral ureteral obstruction. Kidney Int 47: 1285-1294, 1995. 
15. Radović N, Cuzić S and Knotek M: Effect of unilateral ureteral obstruction and anti-angiotensin II treatment on renal tubule and interstitial cell apoptosis in rats. Croat Med J 49: 600-607, 2008

16. Otsuka F, Yamauchi T, Kataoka H, et al: Effects of chronic inhibition of ACE and AT1 receptors on glomerular injury in dahl salt-sensitive rats. Am J Physiol 274: R1797-R1806, 1998.

17. Fujihara CK, Velho M, Malheiros DM and Zatz R: An extremely high dose of losartan affords superior renoprotection in the remnant model. Kidney Int 67: 1913-1924, 2005.

18. Manucha W, Oliveros L, Carrizo L, et al: Losartan modulation on NOS isoforms and COX-2 expression in early renal fibrogenesis in unilateral obstruction. Kidney Int 65: 2091-2107, 2004.

19. Kellner D, Chen J, Richardson I, et al: Angiotensin receptor blockade decreases fibrosis and fibroblast expression in a rat model of unilateral ureteral obstruction. J Urol 176: 806-812, 2006.

20. Eskild-Jensen A, Paulsen LF, Wogensen L, et al: AT1 receptor blockade prevents interstitial and glomerular apoptosis but not fibrosis in pigs with neonatal induced partial unilateral ureteral obstruction. Am J Physiol Renal Physiol 292: F1771-F1781, 2007.
21. Roxburgh SA, Murphy M, Pollock CA and Brazil DP: Recapitulation of embryological programmes in renal fibrosis - the importance of epithelial cell plasticity and developmental genes. Nephron Physiol 103: p139-p148, 2006.

22. Chuang PY and He JC: JAK/STAT signaling in renal diseases. Kidney Int 78: 231-234, 2010.

23. Pang M, Ma L, Gong R, et al: A novel STAT3 inhibitor, S3I-201, attenuates renal interstitial fibroblast activation and interstitial fibrosis in obstructive nephropathy. Kidney Int 78: 257-268, 2010.

24. Matsuda H, Mori T, Kurumazuka D, et al: Inhibitory effects of T/L-type calcium channel blockers on tubulointerstitial fibrosis in obstructed kidneys in rats. Urology 77: 249.e9-15, 2011.

25. Pang M, Kothapally J, Mao $\mathrm{H}$, et al: Inhibition of histone deacetylase activity attenuates renal fibroblast activation and interstitial fibrosis in obstructive nephropathy. Am J Physiol Renal Physiol 297: F996-F1005, 2009.

26. Yang J, Dai C and Liu Y: Hepatocyte growth factor gene therapy and angiotensin II blockade synergistically attenuate renal interstitial fibrosis in mice. J Am Soc Nephrol 13: 2464-2477, 2002.

27. Liu N, Tolbert E, Ponnusamy M, et al: Delayed administration of suramin attenuates the progression of renal fibrosis in obstructive nephropathy. J Pharmacol Exp Ther 338: 758-766, 2011. 